\title{
10.22402/j.rdipycs.unam.7.2.2021.360.330-346
}

\section{ANÁLISIS DE LA EJECUCIÓN Y PERCEPCIÓN AL HABLAR EN PÚBLICO EN UNIVERSITARIOS}

\author{
María Cristina Bravo González, Patricia Ortega Silva y José Esteban Vaquero Cázares \\ Facultad de Estudios Superiores Iztacala, UNAM \\ México
}

\begin{abstract}
RESUMEN
La habilidad para hablar en público es fundamental para todo universitario, incluye comportamientos verbales y no verbales que no siempre son consistentes con la ejecución al hablar en público. El objetivo fue examinar la relación entre la percepción de hablar en público y la observación de la ejecución al exponer. Participaron 41 estudiantes de licenciatura; se utilizó el Cuestionario de Confianza para Hablar en Público y la Rúbrica de Habilidades para Hablar en Público en Estudiantes Universitarios para evaluar la ejecución. Los hallazgos concuerdan con investigaciones previas acerca de la prevalencia de dificultades para hablar en público en universitarios, al ubicarla en 20 y $30 \%$. Pese a que no se encontró una relación significativa entre la percepción y la ejecución al hablar en público, es necesario continuar con la línea de investigación derivada de las posibles combinaciones de percepción-ejecución, con el objetivo de ofrecer entrenamientos diferenciados para mejorar esta habilidad.
\end{abstract}

Palabras Clave:

habilidades, hablar en público, percepción, ejecución, estudiantes universitarios

\section{ANALYSIS OF PERFORMANCE AND PERCEPTION WHEN SPEAKING IN PUBLIC IN UNIVERSITY STUDENTS}

\begin{abstract}
Public speaking skills are fundamental for all university students, and include verbal and nonverbal behaviors that are not always consistent with the performance when speaking in public. The objective was to examine the relationship between perception about in public speaking and observation of performance when exhibiting. Forthy-one undergraduate students participated; the Public Speaking Confidence Questionnaire and the Public Speaking Skills in College Students Rubric were used to assess performance. The findings are consistent with previous research on the prevalence of difficulties in public speaking in college, placing it at $20 \%$ and $30 \%$. Although no significant relationship was found between perception and execution when speaking in public, it is necessary to continue with the line of research derived from the possible combinations of perception-execution, in order to offer differentiated training to improve this skill.
\end{abstract}

Keywords:

skills, public speaking, perception, performance, university students

Bitácora del ARtículo:

| Recibido: 21 de Abril de 2021 | Aceptado: 21 de Mayo de 2021 | Publicado en línea: Julio-Diciembre de 2021 | 
Artículo Empírico | Análisis de la ejecución y percepción al hablar...| Bravo-González, Ortega-Silva eq Vaquero-Cázares

\title{
Autoría y Derechos de Propiedad Intelectual
}

\section{ANÁLISIS DE LA EJECUCIÓN Y PERCEPCIÓN AL HABLAR EN PÚBLICO EN UNIVERSITARIOS}

\author{
María Cristina Bravo González, Patricia Ortega Silva y José Esteban Vaquero Cázares \\ Facultad de Estudios Superiores Iztacala, UNAM \\ México
}

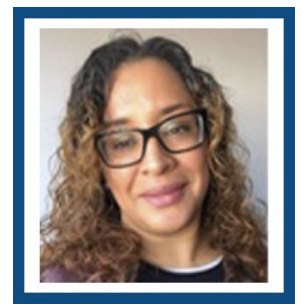

María Cristina Bravo González

FES- Iztacala, UNAM

Correo: cristina.bravo@iztacala.unam.mx

Doctora en Psicología por la Universidad Nacional Autónoma de México, Profesora Asociada "C" de tiempo completo. Tutora, supervisora y docente en la residencia en Medicina conductual, perteneciente al programa de Maestría en Psicología Profesional, UNAM. Profesora en el área de Psicología Clínica en la FESI entre 2004 y 2011; profesora de Metodología en Salud a nivel licenciatura desde 2019 en la FESI.

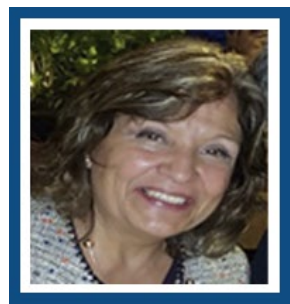

Patricia Ortega Silva FES- Iztacala, UNAM Correo:patosi75@gmail.com

Doctorado en Sociología. Facultad de Ciencias Políticas y Sociales por la Universidad Nacional Autónoma de México. Profesora de la carrera de Psicología, Área de Educación Especial y Rehabilitación en la FES. Iztacala. Profesor titular "C" definitivo tiempo completo. Integrante del padrón de tutores del doctorado y maestría en Psicología. Evaluador de proyectos de investigación propuestos por CONACyT.

\section{CONTRIBUCIÓN DE LOS AUTORES}

María Cristina Bravo González, concibió la idea y participó en la elaboración de todos los apartados del artículo. | Patricia Ortega Silva, participó en la discusión de los argumentos centrales a lo largo de todo el trabajo, lectura final del texto y corrección de estilo. | José Esteban Vaquero Cázares, colaboró en el planteamiento metodológico, el análisis de datos y corrección de estilo.

\section{AGRADECIMIENTOS}

Agradecimiento al Programa de Maestría y Doctorado en Psicología de la UNAM, este trabajo es una parte de la tesis doctoral de la primera autora.

\section{DATOS DE Filiación de los Autores}

Facultad de Estudios Superiores Iztacala, UNAM

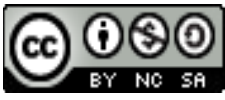

Copyright: (c) 2021 Bravo-González, M.C.; Ortega-Silva, P.; y Vaquero-Cázares, J. E.

Este es un artículo de acceso abierto distribuido bajo los términos de la licencia Creative Commons Reconocimiento-NoComercial 4.0 Internacional, por lo que su contenido gráfico y escrito se puede compartir, copiar y redistribuir total o parcialmente sin necesidad de permiso expreso de sus autoras con la única condición de que no se puede usar con fines directamente comerciales y los términos legales de cualquier trabajo derivado deben ser los mismos que se expresan en la presente declaración. La única condición es que se cite la fuente con referencia a la Revista Digital Internacional de Psicología y Ciencia Social y a sus autores. 


\section{TABLA DE CONTENIDO}

INTRODUCCIÓN

MÉTODO

Participantes, 334

Materiales, 334

Procedimiento

Mediciones, 335

ANÁLISIS ESTADÍSTICOS

Resultados

Discusión

CONCLUSIONES

REFERENCIAS 


\section{INTRODUCCIÓN}

A lo largo de la vida académica, los contenidos a los que se dedica una elevada proporción del tiempo del docente, desde la educación básica hasta la educación superior, enfatizan la necesidad de formar primero y exhibir después "productos" de dos tipos de comunicación; por un lado está la comunicación escrita, la cual se traduce en diversas maneras de expresar el lenguaje mediante diversas estrategias, tanto para leer como para escribir (conocimientos de gramática, ortografía, léxico, sintaxis y redacción), y por otro lado se encuentra la comunicación oral (elementos del diálogo, narrativa, descripción y exposición), la cual implica hacer uso del habla como elemento principal para lograr la expresión de ideas por medio del lenguaje.

Pese al tiempo que se dedica a la formación de estas habilidades, sobre todo en la educación básica, durante la formación universitaria se espera que el estudiante cuente con las estrategias relacionadas con ambos tipos de comunicación: que sea un lector con adecuada comprensión, que haya desarrollado habilidades de análisis y síntesis de los textos, que pueda expresar por escrito su opinión, reflexión o crítica respecto de lo que lee y que lo haga de acuerdo con las reglas en las que fue formado en niveles previos; de manera similar, en la comunicación oral a nivel universitario se espera que el estudiante tenga habilidades avanzadas para expresarse de modo verbal mediante preguntas, describiendo eventos o exponiendo; sin embargo, en las aulas universitarias y en muchos profesionistas ninguna de estas clases de comunicación están bien establecidas (Diafani y Syahrul, 2018; Qadhi, 2018) ni se establecen espacios para su instrucción, porque, de acuerdo con García-López, Díez-Bedmar y Almansa-Moreno (2013), la enseñanza de estas habilidades requiere una gran inversión de tiempo, por lo que esas posibles deficiencias formativas quedan sin corrección para la mayoría de los estudiantes.

En la investigación de Stein, Torgrud y Walker (2000) la mayoría de las personas diagnosticadas con fobia social refirieron que esta problemática ha interferido de manera importante en diversas áreas de su vida; en el ámbito educativo $22 \%$ afirma que ha obstruido su educación, y $48 \%$ indica que ha abandonado clases por esta condición; respecto a otras áreas de funcionamiento, 20\% afir- ma que ha afectado mucho tanto su trabajo como su vida personal, y otras investigaciones aportan datos similares (Randelovic, Smederevac, Colovic y Corr, 2018).

La falta de habilidades para hablar en público puede tener diversas consecuencias para los estudiantes; en primer lugar, en el contexto académico, las actividades de evaluación (exámenes y presentaciones orales) son de los problemas más comunes en los jóvenes universitarios (Bartholomay y Houlihan, 2016), lo que trae como consecuencia problemas en las evaluaciones académicas, porque en la mayoría de las asignaturas las participaciones en clase y la exposición de temas ante el grupo tienen mucha importancia, lo cual puede generar problemas en el rendimiento académico (Morales y Navarro, 2013), por lo que el aprendizaje se ve comprometido (Amiri y Puteh, 2018). Asimismo, Contreras, Espinosa, Esguerra, Haikal y Polanía (2005) indican que la ansiedad aumenta de manera considerable en situaciones de desempeño y evaluación, es decir, se incrementa cuando las consecuencias son importantes (como reprobar una asignatura), por lo que en los individuos con miedo a hablar en público (MHP) pueden encontrarse dificultades académicas importantes (Munz y Colvin, 2018; Qadhi, 2018).

Otra repercusión del problema se sitúa en el plano del sufrimiento personal por la ansiedad experimentada ante esta situación, que en los casos más extremos puede derivar en problemas de salud y la deserción escolar (Moore, Gómez-Ariza y García-López, 2016). En este sentido, según Haquin, Larraguibel y Cabezas (2004), el ambiente escolar es una fuente potencial de satisfacciones para los jóvenes, siempre y cuando pueda desarrollar, ejercitar y practicar sus habilidades, con lo que se incrementa su percepción de control de sí mismo y su entorno, además de aumentar su autoeficacia percibida; cuando ocurre lo contrario y se llega al fracaso o deserción escolar, Contreras et al., (2005) afirman que es un problema que genera exclusiones que serán decisivas en la vida de una persona.

Tener estas habilidades (o competencias sociales) es necesario para un desempeño adecuado en la vida profesional y laboral; es cierto que el éxito profesional se asocia con el conocimiento y habilidades específicas para detectar y saber cómo resolver problemas, pero también se reconoce que un profesional exitoso necesita además una adecuada formación técnica (en su campo de conocimiento) y comunicarse de manera clara y eficaz (Verano-Tacoronte, González-Betancor, Bolívar-Cruz, Fernández-Monroy y Galván-Sánchez, 2016), por lo que debe tener las competencias adecuadas para comunicarse de modo oral y por escrito, entre otras.

Schreiber, Paul y Shibley (2012) consideran que la Asociación Americana de Preparatorias y Universidades 
(Association of American Colleges and Universities, AAC\&U) ha planteado un conjunto de aprendizajes necesarios en la educación superior, y la comunicación oral se incluye en la categoría de "Habilidades intelectuales y prácticas"; además destacan que este tipo de habilidades son esenciales para el desarrollo intelectual; según Morales y Navarro (2013), tener la habilidad para la expresión y comunicación oral es muy importante para lograr un buen desempeño profesional.

Calero (2009) considera la comunicación como una capacidad que se desglosa en una serie de destrezas, como exponer, opinar y explicar (Munz y Colvin, 2018). Por tanto, exponer frente a una clase puede ser considerado un contenido procedimental que implica un conjunto de acciones que se dirigen al logro de un objetivo, es decir, implica emprender acciones, lo cual requiere la realización de éstas; es decir, no puede aprenderse a hablar en público o exponer en una clase de manera teórica (leyendo libros al respecto), se aprende por medio de la repetición de acciones, esto es, mediante la práctica retroalimentada, ejecución comprensiva y contextualizada, que en su conjunto se caracterizan como acciones complejas (Morán, 2018). El aprendizaje procedimental implica el dominio de habilidades o destrezas necesarias para desarrollar bien diversas acciones; en este caso el manejo de técnicas y estrategias para transmitir contenidos de manera verbal.

Pese a la atención que ha recibido el estudio de "Hablar en público", hay una limitación importante respecto a la evaluación, porque los medios utilizados para detectar esta problemática por lo común se restringen a instrumentos de lápiz y papel o mediante entrevista con expertos (en general del ámbito clínico, al considerarse como parte de un subtipo de ansiedad social), y sólo unos cuántos verifican - por medio de la observación directa- el desempeño de esta habilidad, ya sea como parte de un entrenamiento (García-López et al., 2013; Liao, 2014) o como parte de la evaluación de habilidades (Schreiber et al., 2012). Esta condición es peligrosa porque un estudiante puede tener sesgos en su comportamiento que puede resultar en alguna de las siguientes combinaciones: tener puntuaciones que indican dificultades al hablar en público y no exponer de modo adecuado; tener puntuaciones que indican dificultades al hablar en público, pero exponer de manera adecuada; tener puntuaciones que no indican dificultades al hablar en público, pero no exponer de modo adecuado, y tener puntuaciones que no indican dificultades al hablar en público y exponer de manera adecuada. Es decir, los resultados en una prueba autoaplicable pueden no reflejar el desempeño del estudiante; por tanto, el propósito es caracterizar la eje- cución al hablar en público de estudiantes universitarios a partir de la observación del comportamiento durante actividades cotidianas de exposición en una asignatura y el contraste de dicha observación con la percepción de los estudiantes respecto a su habilidad para hablar en público de acuerdo con las calificaciones obtenidas en un instrumento de lápiz y papel.

\section{Método}

\section{Participantes}

Se seleccionó un grupo de alumnos inscritos en el quinto semestre de licenciatura de la carrera de Psicología de la FES Iztacala, mediante un muestreo no probabilístico intencional por conveniencia; el primer criterio para seleccionarlos fue contar con la participación de uno de los profesores de asignaturas teóricas que tuviera en su programa de actividades exposiciones a lo largo del semestre. Además, los integrantes del grupo firmaron un consentimiento con información para autorizar su participación.

El grupo seleccionado a partir de los criterios anteriores fue uno del turno vespertino -41 alumnos, 15 varones y 26 mujeres, con edad promedio de 21.4 años $(\mathrm{DE}=2.2)$ - la asignatura donde se hizo la grabación de cada una de las exposiciones orales fue Psicología Clínica Teórica, con un horario de una hora durante tres días a la semana.

\section{Materiales}

Se diseñó una ficha de datos demográficos y académicos (incluyendo el promedio) y número de asignaturas reprobadas al momento de la investigación, entre otros datos. Se usó el Cuestionario de Confianza para Hablar en Público (CCHP), diseñado por Gilkinson (1942), que Paul modificó en 1966 (citados en Gallego, Botella, Quero, Baños y García-Palacios, 2007), reduciéndose de los 101 ítems originales a 30 ítems con un formato verdadero-falso. En España, Bados (1992, citado en Bados, 2005) modificó el formato de respuesta incorporando una escala tipo Likert de 1 a 6 puntos (de 1: "Completamente de acuerdo", a 6: "Completamente en desacuerdo"), y evalúa las respuestas afectivas, cognitivas y conductuales del miedo a hablar en público. Los niveles pueden variar de 30 a 180 puntos, en los que a mayor puntaje mayor miedo; la puntuación media es de 105 . Tiene 17 reactivos negativos que deben transformarse previo a la obtención de la puntuación total; dichos reactivos son: 2 , 3, 5, 6, 7, 8, 13, 15, 18, 19, 20, $24,25,26,28,29$ y 30 . En un estudio con una muestra 
de 243 estudiantes universitarios mexicanos, se identificó un coeficiente de consistencia interna de 0.93 (Tron, Bravo y Vaquero, 2013), por lo que se concluye que es un cuestionario útil para evaluar el MHP en estudiantes mexicanos de nivel medio y superior.

Además se utilizó la Rúbrica de Habilidades para Hablar en Público en Estudiantes Universitarios (RHHPEU), cuyas características se presentan en un estudio previo (Bravo y Ortega, 2019) y corresponde a la adaptación de la Public Speaking Competence Rubric (PSCR, por sus siglas en inglés) de Schreiber et al., (2012). Se trata de una rúbrica analítica que consta de nueve áreas a evaluar: presentación, organización, preparación, lenguaje, dicción, expresión verbal, conductas no verbales, apoyos visuales y conclusión; las cuales se valoran del 0 al 4 , donde 0 corresponde a una habilidad deficiente y 4 a una habilidad avanzada.

Se utilizó un teléfono celular marca iPhone $8 \circledR$ de 62 GB de capacidad, audífonos marca Apple ${ }^{\circledR}$ con función de micrófono y tripié con adaptador para teléfono celular, para videograbar las exposiciones. Por último se usaron formatos de registro en Excel ${ }^{\circledR}$ para completar la RHHPEU para evaluar a cada participante.

\section{Procedimiento}

Se acudió al salón de clase en el horario asignado para la materia en cuestión, con la participación del profesor a cargo, quien presentó a la investigadora responsable y comentó en términos generales el objetivo del estudio. Después se explicó de manera minuciosa la finalidad de la investigación, así como el tipo de actividades que se desarrollarían en caso de aceptar; luego de la explicación se entregó un formato de consentimiento informado a cada estudiante, el cual se elaboró considerando los lineamientos establecidos por la Facultad de Estudios Superiores Iztacala; se indicó que la participación en la investigación implicaba un riesgo mínimo, así como la garantía de anonimato, confidencialidad y uso de datos personales. Se solicitó a los estudiantes que leyeran con detenimiento el consentimiento, y si estaban de acuerdo en participar completaran sus datos y lo firmaran.

Una vez que los integrantes del grupo leyeron y firmaron el consentimiento informado se procedió a la aplicación del Cuestionario Confianza para Hablar en Público; al término se estableció la fecha de inicio de las exposiciones de acuerdo con el plan de actividades y organización prevista por el profesor. Después se asistió a todas las clases donde se tuvo programada la exposición oral de un tema por un estudiante para su grabación; en cada ocasión se procuró mantener la misma ubicación (centro del aula, última fila). Se estableció que cada grabación iniciara unos segundos antes de que comenzara la intervención del estudiante, y en general se mantuvo hasta el final de la clase, por lo que se incluyeron los comentarios del profesor, las participaciones, preguntas o comentarios de la audiencia.

Cada exposición fue observada y evaluada por tres observadores entrenados ex profeso en el llenado de la RHHPEU, en un momento posterior a la clase y de manera independiente, porque se almacenaron en carpetas separadas con el uso de la plataforma Dropbox.

\section{Mediciones}

En este estudio se evaluaron dos variables: la ejecución al hablar en público, que se define como un conjunto de comportamientos específicos verbales y no verbales, que se desarrolla de acuerdo con el contexto (Caballo, 1993), que se operacionalizó con la correlación del acuerdo entre observadores por medio del coeficiente kappa de Krippendorf derivado del Ilenado de la RHHPEU y la determinación del nivel de ejecución.

Por otro lado se evalúo la percepción de la habilidad para hablar en público, que se caracterizó como la valoración subjetiva de una persona respecto de sus habilidades al hablar en público, la cual se operacionalizó con el índice obtenido con el Cuestionario de Confianza para Hablar en Público, donde a mayor puntuación, menor confianza.

Durante la investigación no se tuvo control de variables extrañas que pudieron tener una función importante en la percepción de las habilidades o en la ejecución al exponer en clase, como estados afectivos o emocionales que pudieran interferir con el desempeño, entre otros.

\section{Análisis estadísticos}

Los análisis estadísticos incluyen medidas de distribución porcentual y el cálculo de medidas de tendencia central para describir las características de los participantes, así como aspectos relacionados con la actividad de hablar en público en contextos académicos.

Para determinar la confiabilidad de las observaciones registradas en la Rúbrica de Habilidades para Hablar en Público en Estudiantes Universitarios (RHHPEU), se calculó el alfa de Krippendorff ( $\boldsymbol{\alpha}, \mathrm{KALPHA})$, que es un coeficiente de confiabilidad desarrollado para medir el acuerdo entre observadores y es aplicable a dos o más métodos para generar datos en un mismo conjunto de objetos, unidades de análisis o reactivos (Krippendorff, 2011); este coeficiente se puede aplicar a cualquier número de observadores y/o a cualquier número de catego- 
rías o valores en una escala; puede usarse con cualquier escala de medición, así como en el caso de que existan valores perdidos, y no requiere un tamaño mínimo de muestra (De Swert, 2012), razones por las que se determinó que era el más apropiado para analizar las observaciones obtenidas a partir de la rúbrica.

Por último, se calculó el coeficiente de correlación rho de Spearman para examinar la relación entre el nivel de ejecución y la percepción al hablar en público; según Field (2018), este coeficiente es apropiado para analizar la relación entre dos variables, donde al menos una de ellas se encuentra en un nivel de medición ordinal, como el caso del nivel de ejecución al hablar en público.

\section{Resultados}

Se encontró que $46 \%$ de los participantes ingresó a la licenciatura con un promedio entre 8 y $8.9 ; 44 \%$ con un promedio entre 7 y $7.9 ; 8 \%$ de los participantes con un promedio de 9 o más, y sólo $2 \%$ con un promedio inferior a 7. Respecto al promedio obtenido por los participantes en la carrera hasta el momento de la investigación, 54\% de los estudiantes tiene un promedio entre 8 y $8.9 ; 31 \%$ tiene una calificación promedio entre 7 y 7.9 , mientras que $14 \%$ tiene un promedio de al menos 9 de calificación. Aunque $54 \%$ de los estudiantes es un alumno regular, casi la mitad (46\%) de los participantes tiene asignaturas reprobadas; de ellos, la mayoría tiene sólo una materia no aprobada (54\%), $17 \%$ tiene dos y $29 \%$ de los participantes no ha aprobado cinco o más asignaturas.

Ante la pregunta explícita de tener dificultades para participar o exponer en clase, 68\% respondió de modo afirmativo. Los participantes indicaron algunas razones por las que, en su opinión, les resulta difícil participar o exponer en clase; las respuestas se agruparon en nueve categorías debido a que la pregunta tiene un carácter abierto; en la figura 1 se muestra la distribución porcentual de las razones que los participantes indicaron como causas de sus dificultades para participar y/o exponer en clase.

Como puede observarse, poco más de $25 \%$ de los participantes aludió a situaciones relacionadas con ansiedad o, en sus propias palabras, "sentir nervios", "pánico escénico", "pena de exponer ideas propias" e "inseguridad", entre otras; la segunda categoría con más menciones se refiere a cogniciones relacionadas con temor a equivocarse (como miedo a que la participación no sea buena, miedo a no explicar bien el tema, miedo a ser corregido, etcétera); la tercera razón mencionada se refiere a no dominar el tema o prepararlo de modo inadecuado. El resto de las categorías agrupa razones que tuvieron menos de $10 \%$ de menciones, entre las que se encuentran pensamientos negativos, profesor/compañeros hostiles, olvidar información/bloquearse, perturbaciones del habla, tamaño del grupo y otras razones.

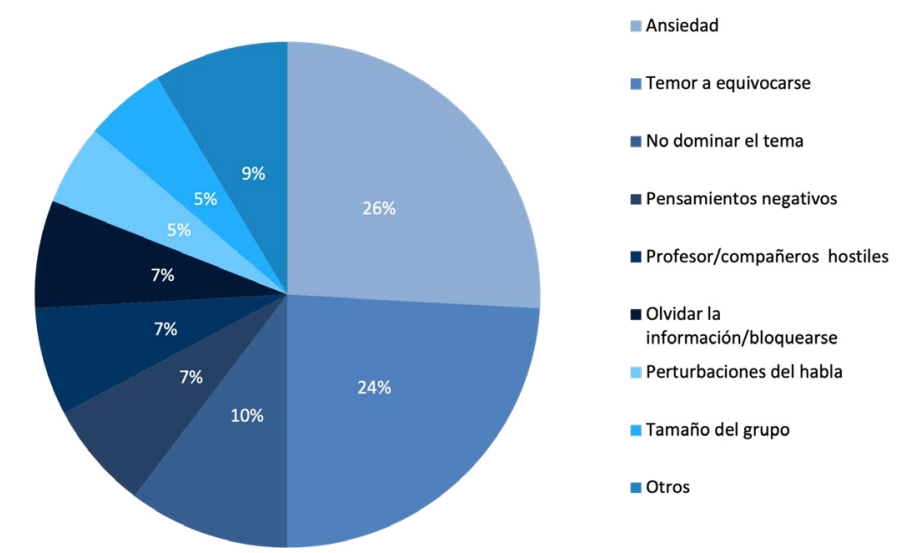

Figura 1.

Distribución porcentual de las razones por las que los participantes refieren dificultades para participar o exponer en clase.

Respecto a la duración de cada exposición, se encontró que $17 \%$ de los participantes tuvo una ejecución muy breve al hablar en público (10 minutos o menos), mientras que $51 \%$ tuvo una exposición de entre 11 y 30 minutos, que aún es breve para exponer un texto académico. Sin embargo, también se identificaron algunos estudiantes que utilizaron 50 minutos o más (12\%).

En el CCHP se identificó un índice promedio de 100 (DE = 25.8), el cual está muy cercano del nivel promedio indicado por Bados (1992, citado en Bados, 2005) en su validación al español, que es de 105; esta puntuación se ubica en un nivel medio de respuestas afectivas, cognitivas y conductuales asociadas a hablar en público. Se obtuvieron los percentiles para el CCHP, de acuerdo con sus niveles mínimo y máximo, a partir de los cuales se encontró que $24 \%$ de los participantes tuvo una puntuación por encima de $75 \%$, lo cual implica que tienen un nivel elevado de miedo a hablar en público, el cual se refleja en las tres clases de respuesta que evalúa el instrumento; por otro lado, $27 \%$ de los participantes evaluados obtuvieron una puntuación por debajo de $25 \%$, es decir, estos estudiantes se sienten confiados al hablar en público. Se analizaron las respuestas de los participantes con niveles altos y bajos en el CCHP considerando el reporte de sus habilidades para hablar en público, donde se encontró que de los estudiantes que obtuvieron niveles bajos, $18 \%$ se percibe con dificultades para participar o exponer en clase, mientras que de los estudiantes con puntuaciones elevadas el 90\% refirió esas mismas dificultades. 
De acuerdo con las observaciones que se consideran confiables a partir del cálculo de KALPHA ( $\mathrm{n}=36)$, se determinó el nivel de habilidad para hablar en público de cada uno de los participantes, considerando la estimación del nivel promedio obtenido en la RHHPEU. En la figura 2 se muestra la distribución porcentual de las habilidades para hablar en público de los estudiantes al exponer un texto. Como se puede observar, en la Rúbrica de Habilidades para Hablar en Público en Estudiantes Universitarios la mitad de los participantes obtuvo un promedio que los ubica en un nivel de habilidad básico, mientras que para $32 \%$ de los participantes se determinó un nivel de habilidad mínimo en su ejecución al exponer. Por otro lado, sólo 14\% obtuvo puntuaciones en la rúbrica que los ubica en un nivel de habilidad competente; sin embargo, Ilama la atención que no se identificó a ninguno de los participantes con habilidades avanzadas al hablar en público, de acuerdo con las áreas que se evaluaron. Sólo 3\% de los estudiantes considerados $(n=$ 36) se identificó con habilidades deficientes para hablar en público. Al analizar cada una de las habilidades que se evalúan en la RHHPEU, se encontró que las áreas que obtienen puntuaciones más bajas son las de presentación (inicio de la exposición) y la de conclusiones; en ésta se observó que 95\% de los estudiantes no concluyó el tema. Respecto a la habilidad donde se obtuvieron mejores índices, se observó en la dicción; en el resto de las áreas la mayoría de los estudiantes muestra un nivel de habilidad básico (figura 3).

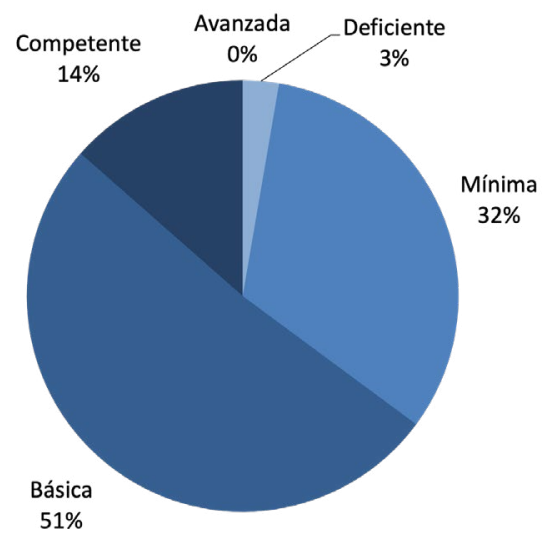

\section{Figura 2.}

Distribución porcentual del nivel de habilidad para hablar en público a partir de Rúbrica de Habilidades para Hablar en Público.

Para analizar la relación entre el autorreporte de los estudiantes universitarios respecto al comportamiento de hablar en público, que se evaluó a partir de las respuestas que proporcionaron al CCHP y a la observación de su ejecución al exponer en clase, evaluada a partir de la rúbrica, se hizo un análisis de correlación no paramétrico con el coeficiente rho de Spearman, porque los datos obtenidos en la rúbrica se encuentran en un nivel ordinal. A partir de este análisis se encontró que el nivel de ejecución determinado a partir de la RHHPEU no correlacionó de manera estadísticamente significativa con el instrumento de autoinforme ( $r s=0.005, p>$ $0.05)$, lo que sugiere que no hay relación entre el nivel de ejecución al hablar en público determinado por observadores externos y la percepción que cada estudiante tiene de su desempeño al hablar en público.

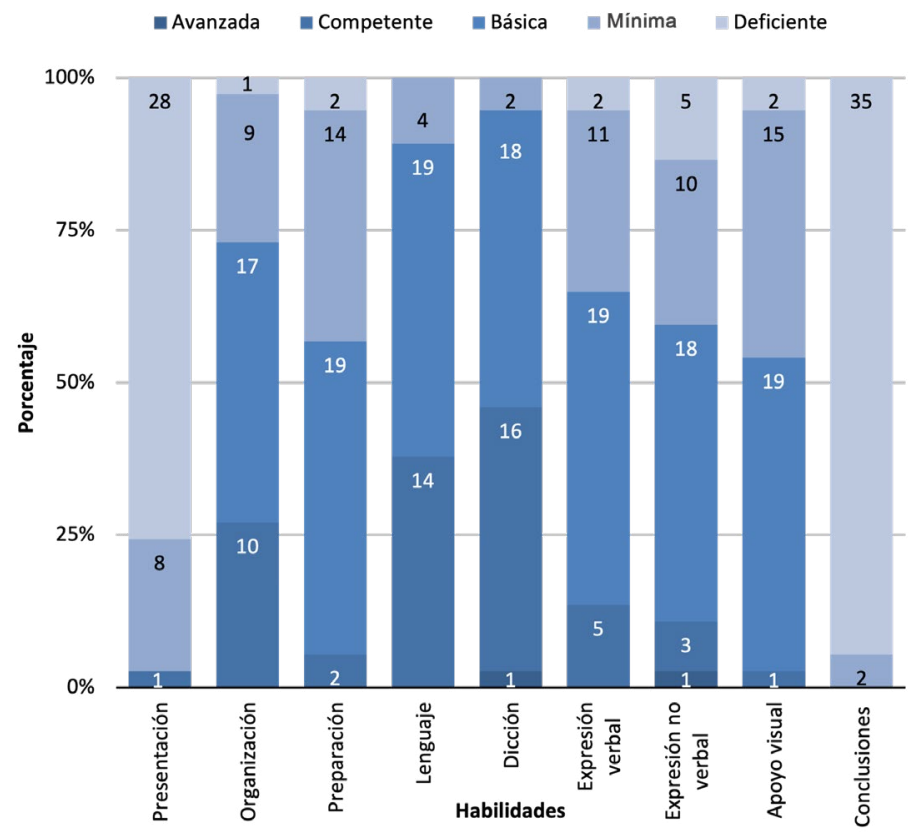

Figura 3.

Distribución porcentual de los niveles de habilidad identificados por área de acuerdo con la RHHPEU.

\section{Discusión}

Martínez (2015) considera que el principal elemento que caracteriza a los seres humanos es el aspecto social, ante lo cual la comunicación es una condición indispensable. Sin embargo los resultados de esta investigación indican que una cantidad considerable de estudiantes tiene dificultades para hablar en público en contextos académicos, lo cual rebasa de manera importante datos mencionados por Hernández-Pozo et al., (2009), quienes indican que en población mexicana general hablar en público es reportado por $24.8 \%$ como muy atemorizante, mientras que $68 \%$ de los participantes en esta investigación refirió tener dificultades en ese rubro; otras investigaciones desarrolladas en otros contextos identifican a la situación de hablar en público como una de 
las situaciones sociales que generan mayor temor, tanto en estudiantes universitarios como en la población general (Bados, 2005; Dornalechete y Domínguez, 2010), aunque en niveles entre 20 y $30 \%$, los cuales están muy alejados de los hallazgos de esta investigación.

$\mathrm{Al}$ analizar las razones por las que los estudiantes refieren tener dificultades para hablar en público, más de $90 \%$ alude a condiciones personales, las cuales incluyen comportamientos, sentimientos y/o cogniciones que dificultan mostrar una ejecución adecuada, porque, de acuerdo con Modini y Abbott (2016), las personas con miedo a hablar en público presentan preocupaciones acerca de su ejecución (como, literalmente, tener pensamientos negativos) y la manera en que pueden ser evaluadas por los demás (manifestadas en esta investigación como temor a equivocarse), lo cual con frecuencia impide procesar la información de manera adecuada (como olvidar la información) y, en consecuencia, hay un detrimento en la actuación del expositor (referido por los participantes como no dominar el tema). Además, según Contreras et al., (2005), las personas presentan niveles de ansiedad diferenciados en situaciones sociales particulares; esto se representa en las variaciones de los temas en torno a los cuales se atribuyen dificultades para lograr una ejecución al hablar en público satisfactoria; esto implica que cada persona tiene razones diferentes ante las cuales se siente atemorizada en situaciones que necesita hablar en público, algunas referidas a las creencias que tienen de los demás, las creencias respecto de su propia actuación o las creencias de las consecuencias que tendrán si su desempeño es evaluado como deficiente.

De los datos obtenidos a partir del CCHP se encontraron aspectos similares a los reportados en otras investigaciones respecto a la prevalencia del miedo a hablar en público tanto en universitarios como en la población general; en particular se identificó que entre $24-27 \%$ de los participantes tiene calificaciones elevadas, las cuales indican la presencia de respuestas afectivas, cognitivas y conductuales de miedo a hablar en público (CCHP), mientras que en investigaciones previas la prevalencia de dificultades para hablar en público varía entre 20 (Gültekin y Dereboy, 2011) y 34\% (Stein, Walker y Forde, 1996) en poblaciones universitarias, por citar algunos, por lo que pareciera que esta condición está presente en una tercera parte de los estudiantes universitarios.

El objetivo general de este trabajo es analizar la relación entre el autorreporte de los estudiantes universitarios acerca del comportamiento de hablar en público y la observación de su ejecución al exponer en clase, y se estableció la hipótesis de que los estudiantes que obtuvieran los índices más altos en el CCHP, es decir, en quienes se identificara miedo a hablar en público por puntuación tendrían un nivel de ejecución deficiente o mínimo; sin embargo, los hallazgos fueron diferentes a los esperados, es decir, se encontraron diversas combinaciones de resultados, aunque no fueron estadísticamente significativas: estudiantes que se perciben con temor a hablar en público, pero que su nivel de ejecución es básico, e incluso en algunos casos competente, lo cual, de acuerdo con Hofmann (2007), puede explicarse porque los sesgos atencionales pueden favorecer que se sobreestimen las consecuencias negativas (Randelovic et al., 2018) de la situación social, se perciben con escaso control en sus emociones y consideran que sus habilidades son inadecuadas o insuficientes.

Por otro lado se identificaron estudiantes cuyos índices reflejan ausencia de miedo a hablar en público; sin embargo, los resultados de la observación de su desempeño al exponer muestran que la mayoría de estos participantes tiene habilidades mínimas, es decir, que su desempeño fue incluso menor que el de participantes identificados con niveles altos para ansiedad social y miedo a hablar en público; esto pudiera deberse a que se trata de personas que no tienen dificultades en ninguna otra situación social, y por consiguiente minimizan las posibles dificultades que puedan llegar a ocurrirles en relación con hablar en público. Lo anterior puede explicarse porque el adulto joven, igual que el adolescente, puede no percatarse de la discrepancia que puede existir entre cómo se ve a sí mismo y cómo piensa o cree que lo ven los demás (Olivares, Rosa y García-López, 2004).

\section{Conclusiones}

De acuerdo con Caballo, Del Prette, Monjas y Carrillo (2016), hablar en público hace referencia a un tipo de conducta interpersonal que consiste en un conjunto de capacidades de actuación y ejecución que tienen un carácter aprendido, por lo que todos los estudiantes, según su habilidad, pueden verse beneficiados de un entrenamiento apropiado a sus características (Mörtberg, Jansson-Fröjmark, Pettersson y Hennlid-Oredsoon, 2018; Munz y Colvin, 2018). Esto es esclarecedor al revisar los hallazgos respecto del nivel de habilidad en la ejecución de los participantes, donde destaca que la mitad de ellos se encuentran en un nivel básico, cuando se esperaría que los estudiantes universitarios que están a la mitad de su carrera tendrían una ejecución mucho más competente e incluso avanzada.

Esta condición no puede explicarse por carencia de práctica, porque en las diversas asignaturas que se 
imparten en la carrera de Psicología de la FES Iztacala, una de las actividades que se desarrolla con mayor frecuencia es la exposición oral de un tema ante el grupo; sin embargo, pese a la idea muy difundida de que "para hablar bien" en público no hay más que practicar lo más frecuentemente posible (Zúñiga, 2012), todo lo contrario, la habilidad requiere ser formada, practicada y retroalimentada (Morán, 2018), de preferencia desde edades tempranas.

Los hallazgos de esta investigación sugieren que la percepción que un individuo puede tener sobre sí mismo y su comportamiento no necesariamente son consistentes con la ejecución que puede mostrar en las situaciones reales, o que tendría que diseñarse una investigación que permitiera aportar datos al respecto; aunque hay suficiente evidencia respecto de que las personas con ansiedad social (generalizada o no generalizada) tienen mayores dificultades para hablar en público (Caballo, Salazar, Robles, Irurta y Equipo de investigación CISO-A México, 2016; Osório, Crippa y Loureiro, 2008), los resultados de esta investigación parecen indicar que calificar alto en un instrumento que evalúa estas condiciones no representa una diferencia en la ejecución de una habilidad concreta, y que el desempeño que se tiene al hablar en público puede explicarse, en las personas en las que no se identifica algún tipo de ansiedad social, por razones de otra naturaleza, como percepción errónea de las propias habilidades, procesos formativos enfocados en otras habilidades y ausencia de retroalimentación efectiva, entre otros aspectos; por tanto, es una línea de investigación a seguir en el futuro, en la cual será imprescindible la incorporación de nuevas tecnologías que favorezcan la mejoría en estas habilidades, como sugieren Diafani y Syahrul (2018), por medio de aplicaciones como Instagram y Facebook (Valerio y Serna, 2018) y con el apoyo de dispositivos móviles como refieren Shamsi, Altaha y Gilanlioglu (2019).

\section{Referencias}

Amiri, F., \& Puteh, M. (2018). Oral Communication Apprehension among International Doctoral Students. English Language Teaching, 11(2), 164-171. http://doi.org/10.5539/elt. v1 1 n $2 \mathrm{p} 164$

Bados, A. (2005). Miedo a hablar en público. Barcelona: Universidad de Barcelona.

Bartholomay, E., \& Houlihan, D. (2016). Public Speaking Anxiety Scale: Preliminary psychometric data and scale validation. Personality and Individual Differences, 94, 211-215. http://dx.doi.org/10.1016/j.paid.2016.01.026

Bravo, M., \& Ortega, P. (2019). Traducción y adaptación de la Public Speaking Competence Rubric como una herramienta para evaluar presentaciones orales. Revista de Educación y Desarrollo, 49, 57-64.

Caballo, V. E. (1993). Manual de evaluación y entrenamiento de las habilidades sociales. Madrid: Siglo XXI.

Caballo, V. E., Del Prette, Z. A. P., Monjas, M. I., \& Carrillo, G. (2016). La evaluación de las habilidades sociales en la vida adulta. En V. E. Caballo (ed.). Manual para la evaluación clínica de los trastornos psicológicos: Trastornos de la edad adulta e informes psicológicos. Madrid: Pirámide.

Caballo, V. E., Salazar, I. C., Robles, R., Irurta, M. J., \& Equipo de investigación CISO-A México (2016). Validación del Cuestionario de Ansiedad Social para Adultos (CASO-A30) en México/Validation of the Social Anxiety Questionnaire for Adults (SAQ) in Mexico. Revista Mexicana de Psicología, 33(1), 30-40. Recuperado de http://www.redalyc.org/ articulo.oa?id $=243056043004$

Calero, M., (2009). Aprendizajes sin límites. Constructivismo. México: Alfaomega.

Contreras, F., Espinosa, J. C., Esguerra, G., Haikal, A., \& Polanía, A. (2005). Autoeficacia, ansiedad y rendimiento académico en adolescentes. Perspectivas en Psicología, 1(2), 183-194. https://doi.org/10.15332/s1794-9998.2005.0002.06

De Swert, K. (2012). Calculating inter-coder reliability in media content analysis using Krippendorff's Alpha. Recuperado de https://www.polcomm.org/wp-content/uploads/ ICR01022012.pdf

Diafiani, S., \& Syahrul, M. (2018). Improving students' public speaking skills through Instagram. Advances in Social Science, Education and Humanities Research, 263, 268271. https://dx.doi.org/10.2991/iclle-18.2018.45

Dornalechete, J., \& Domínguez, A. (2010). Oratoria online: Una propuesta didáctica para la alfabetización digital y la mejora de las competencias comunicativas orales de estudiantes universitarios. Presentado en el II Congreso Internacional Comunicación 3.0, 3 y 4 de octubre. Recuperado de http://campus.usal. es/ comunicacion3punto0/comunicaciones/004.pdf

Field, A. (2018). Discovering statistiscs usign SPSS. 5a. ed. Londres: SAGE.

Gallego, M., Botella, C., Quero, S., Baños, R., \& García-Palacios, A. (2007). Propiedades psicométricas de la escala de miedo a la evaluación negativa versión breve (BFNE) en muestra clínica. Revista de Psicopatología y Psicología Clínica, 12(3), 163-176.

García-López, L., Díez-Bedmar, B., \& Almansa-Moreno, J. (2013). From Being a Trainee to Being a Trainer: Helping Peers Improve their Public Speaking Skills. Revista de Psicodidáctica, 18(2), 331-342. https://dx.doi. org/10.1387/RevPsicodidact.6419

Gültekin, B., \& Dereboy, I. (2011). The prevalence of social phobia, and its impact on quality of life, academic achievement, and identity formation in university students. Turk Psikiyatri Dergisi, 22(3), 150-158. PMID: 21870304.

Haquin, C., Larraguibel, M., \& Cabezas, J. (2004). Factores protectores $\mathrm{y}$ de riesgo en salud mental en niños y adolescentes de la ciudad de Calama. Revista Chilena de Pediatría, 75(5), 425-433. http://dx.doi.org/10.4067/ $\underline{\text { s0370-41062004000500003 }}$ 
Hernández-Pozo, M. R., Calleja, N., Sánchez, A., Coronado, O., Macías, D., \& Cerezo, S. (2009). Validación de una lista de miedos en población mexicana. Acta Colombiana de Psicología, 12(1), 135-150.

Hofmann, S. (2007). Cognitive factors that maintain social anxiety disorder: A comprehensive model and its treatment implications. Cognitive Behaviour Therapy, 36(4), 193209. http://dx.doi.org/10.1080/16506070701421313

Krippendorff, K. (2011). Computing Krippendroff's AlphaReliability. Recuperado de http://repository.upenn.ed/ asc_papers $/ 43$

Liao, H. (2014). Examining the role of collaborative learning in a public speaking course. College Teaching, 62, 47-54. https://doi.org/10.1080/87567555.2013.855891

Martínez, N. (2015). La competencia comunicación interpersonal. En L. Villardón-Gallego (coord.). Competencias genéricas en educación superior. Metodologías específicas para su desarrollo (141-158). Madrid: Narcea.

Modini, M., \& Abbott, M. (2016). A comprehensive review of the cognitive determinants of anxiety and rumination in social anxiety disorder. Behaviour Change, 33(3), 150-171. https://doi.org/10.1017/bec.2016.10

Morales, Z., \& Navarro, Y. (2013). Evaluación de la competencia comunicativa "Hablar en público" en un grupo de universitarios tras un curso sobre habilidades interpersonales. Revista de Enseñanza Universitaria (39), 1-10.

Morán, J. (2018). Evaluar actividades complejas a través de rúbricas. NefroPlus, 10(1), 16-19.

Moore, H., Gómez-Ariza, C., \& García-López, L. (2016). Stopping the past from intruding the present: Social anxiety disorder and proactive interference. Psychiatry Research, 238, 284289. http://dx.doi.org/10.1016/j.psychres.2016.02.053

Mörtberg, E., Jansson-Fröjmark, M., Pettersson, A., \& HennlidOredsoon, T. (2018). Psychometric properties of the Personal Report of Public Speaking Anxiety (PRPSA) in a Sample of University Students in Sweden. International Journal of Cognitive Therapy, 11(4), 421-43. https://doi. org/10.1007/s41811-018-0022-0

Munz, S., \& Colvin, J. (2018). Communication apprehension: Understanding communication skills and cultural identity in the basic communication course. Basic Communication Course Annual, 30, 172-199. https://ecommons.udayton. edu/bcca/vol30/iss1/10

Olivares, J., Rosa, A., \& García-López, L. (2004). Fobia social en la adolescencia. Madrid: Pirámide.

Osório, F., Crippa, J., \& Loureiro, S. (2008). Experimental Models for the Evaluation of Speech and Public Speaking Anxiety:
A Critical Review of the Designs AdoptedThe Journal of Speech and Language Pathology - Applied Behavior Analysis, 3(1), 97-121. http://dx.doi.org/10.1037/ $\underline{\text { h0100236 }}$

Qadhi, A. (2018). Instructional Strategies to Develop the Speaking Skill. International Journal of Linguistics, Literature and Translation, 1(3), 33-36. Recuperado de https://alkindipublisher.com/index.php/ijllt/article/view/317

Randelovic, K., Smederevac, S., Colovic, P., \& Corr, P. (2018). Fear and Anxiety in social setting. Journal of Individual Differences, 39(2). https://doi.org/10.1027/1614$0001 / \mathrm{a} 000251$

Schreiber, L. M., Paul, G. D., \& Shibley, L. R. (2012). The Development and Test of the Public Speaking Competence Rubric. Communication Education, 61(3), 205-233. http:// doi.org/10.1080/03634523.2012.670709

Shamsi, A., Altaha, S., \& Gilanlioglu, I. (2019). The Role of M-Learning in Decreasing Speaking Anxiety for EFL Learners. International Journal of Linguistics, Literature and Translation, 2(1), 276-282. http://doi.org/10.32996/ ijllt.2019.2.1.34

Stein, M., Torgrud, L., \& Walker, J. (2000). Social Phobia Symptoms, Subtypes, and Severity Findings from a Community Survey. Archive General of Psychiatry, 57(1), 1-7. http://doi.org/10.1001/archpsyc.57.11.1046

Stein, M. B., Walker, J. R. \& Forde, I. R. (1996). Public speaking fears in a community sample: Prevalence, impact on functioning, and diagnostic classification. Archives of General Psychiatry, 53, 169-174. http://doi.org/10.1001/ archpsyc.1996.01830020087010

Tron, R., Bravo, C., \& Vaquero, J. (2013). Evaluación de la seguridad para hablar en público en estudiantes universitarios. Cartel presentado en el VI Congreso Latinoamericano de Psicología de la Salud "Bienestar y calidad de vida", del 5 al 7 de junio en San Luis Potosí, México.

Valerio, G., \& Serna, R. (2018). Redes sociales y bienestar psicológico del estudiante universitario. Revista Electrónica de Investigación Educativa, 20(3), 19-28. https://doi.org/10.24320/redie.2018.20.3.1796

Verano-Tacoronte, D., González-Betancor, S., Bolívar-Cruz, A., Fernández-Monroy, M., \& Galván-Sánchez, I. (2016). Valoración de la competencia de comunicación oral de estudiantes universitarios a través de una rúbrica fiable y válida. Revista Brasileira de Educação, 21(64), 39-60. http://dx.doi.org/10.1590/S1413-24782016216403

Zúñiga, H. (2012). Hablar bien en público de una vez por todas. México: Océano. 
Artículo Empírico | Análisis de la ejecución y percepción al hablar... | Bravo-González, Ortega-Silva el Vaquero-Cázares

\section{Meta-Análisis del Artículo}




\section{Dimensión Cuantitativa}

\section{Perfil de Evaluación entre pares}
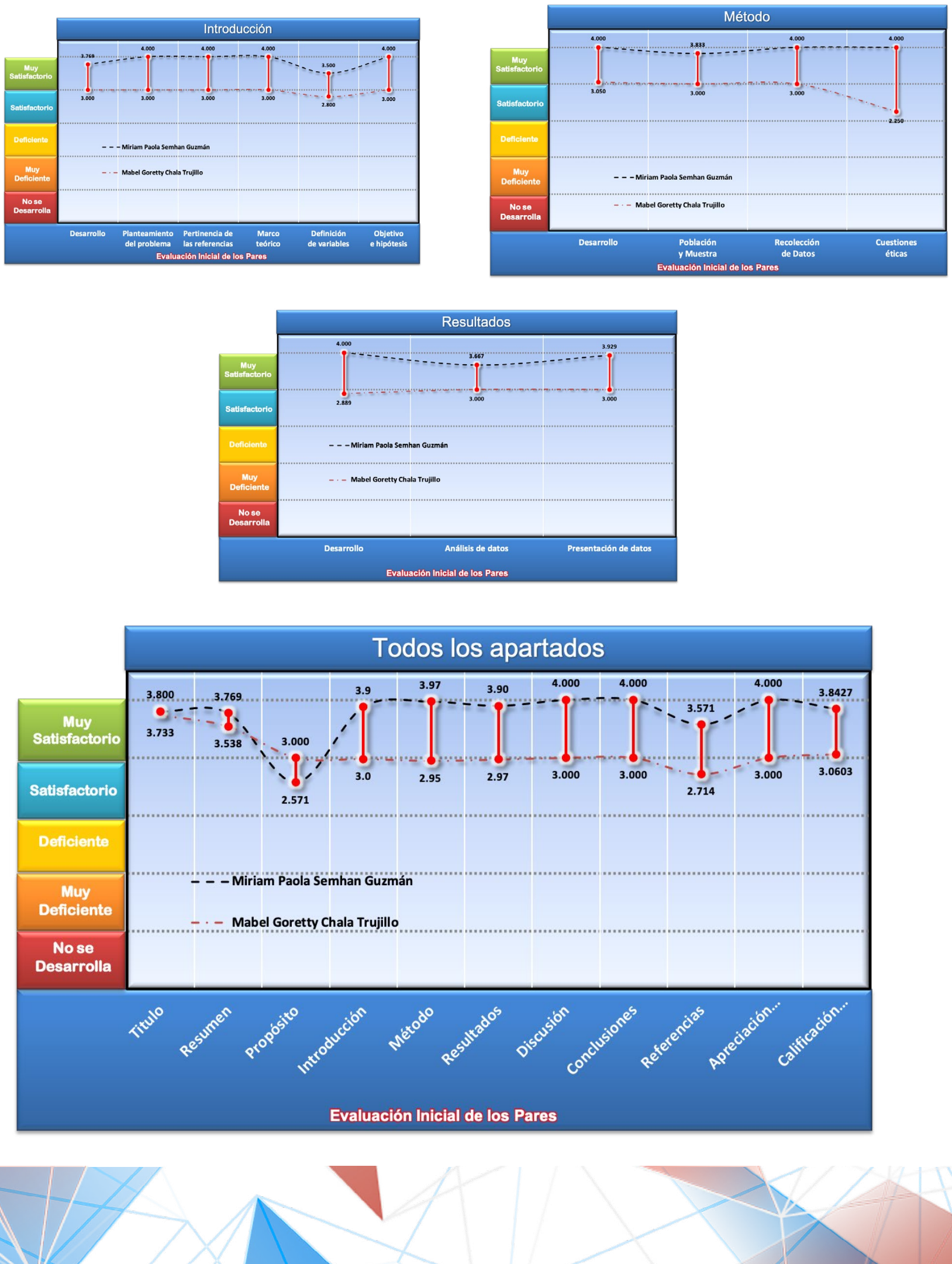


\section{Índice de Concordancia}

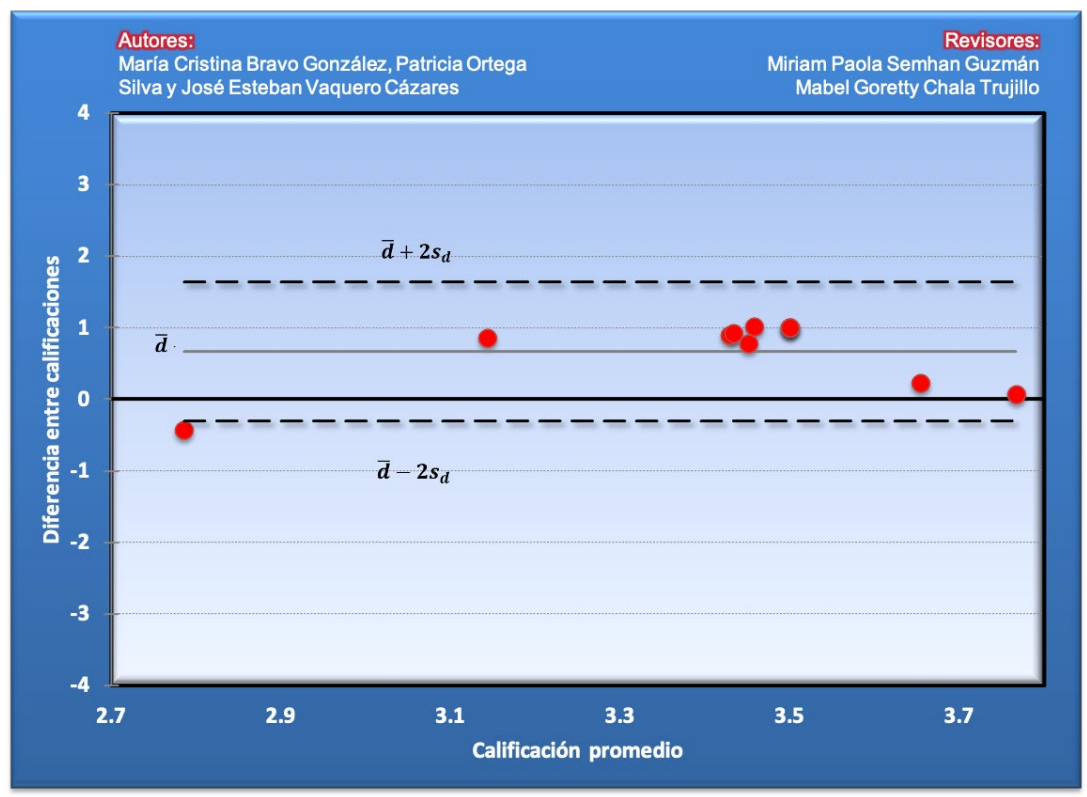

\section{Índice de Acuerdo}

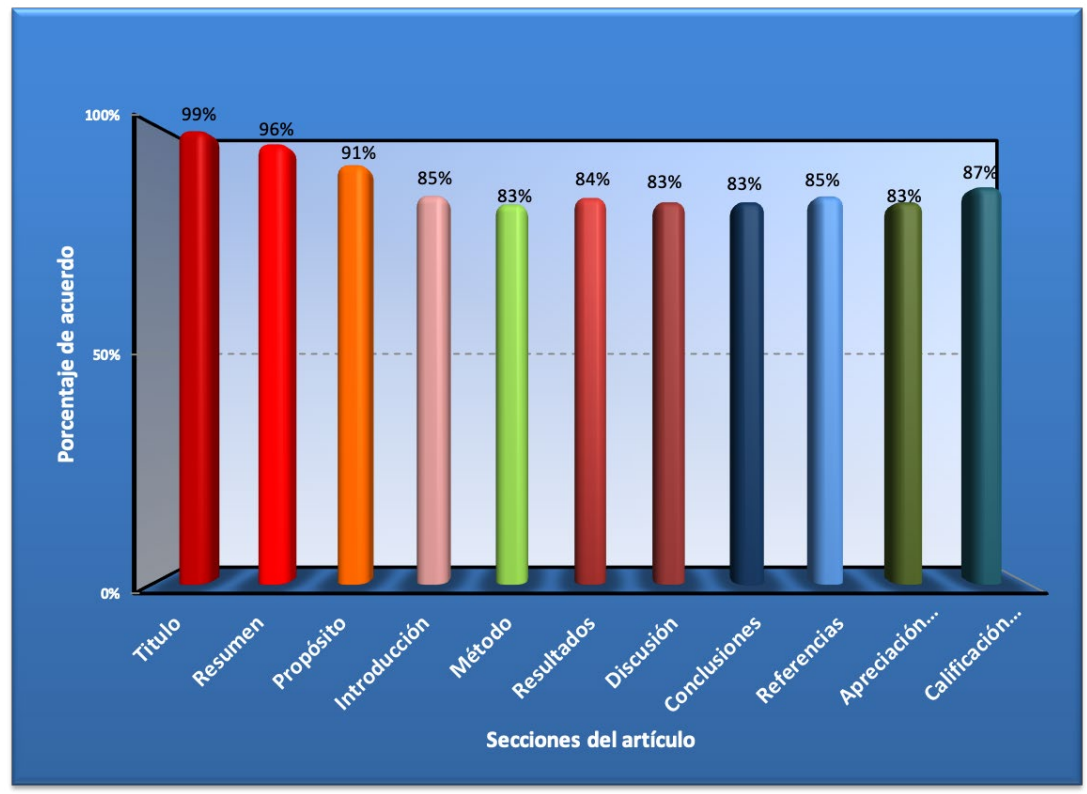




\begin{tabular}{|l|}
\hline \multicolumn{1}{|c|}{ Revisor 1 } \\
\hline \multicolumn{1}{|c|}{ Miriam Paola Semhan Guzman } \\
\hline \multicolumn{2}{|c|}{ Título/A } \\
$\begin{array}{l}\text { En el artículo hablan de percepción y ejecución de la ha- } \\
\text { bilidad, sin embargo en el título solamente se menciona la } \\
\text { ejecución. }\end{array}$
\end{tabular}

\section{Resumen}

El título es adecuado, claro, sencillo. En cuanto a los autores, si bien, se describen los datos de contacto de la autora principal con una nota, faltaría el correo de la autora dos y el correo más la síntesis curricular del autor tres.

En cuanto a contenido es claro y tiene la estructura espe-

El resumen consta de 201 palabras rada. Sin embargo, supera las 150 palabras indicadas, pues cuenta con 201 palabras. Incluye cuatro palabras clave.

\section{Próposito del Estudio}

Aunque el propósito de estudio se entiende, se justifica perfectamente la razón del estudio, sin embargo no se especifican las preguntas de investigación o hipótesis.

Se presentan hipótesis claras y concretas desde las cuales se describen la discusión y los hallazgos del estudio.

\section{Introducción}

Ninguna.

La introducción es clara y señala los aspectos esperados para ubicar al lector en el documento y en lo que pretende la investigación. Se sugiere incluir que no se consideran variables extrañas como el mismo ejercicio de participar en el estudio o estados emocionales o afectivos de los participantes, ajenos a la situación de hablar en público, relacionados con otras dimensiones de la vida, que podrían afectar su desempeño al hablar en público en el escenario evaluado.

\section{Método}

Ninguno

El método está suficiente y claramente descrito. Se sugiere incluir el cumplimiento de la normatividad mexicana inherente a un ejercicio investigativo de riesgo mínimo para los participantes, como es éste. 
Artículo Empírico | Análisis de la ejecución y percepción al hablar... | Bravo-González, Ortega-Silva eL Vaquero-Cázares

\begin{tabular}{|c|c|}
\hline Revisor 1 & Revisor 2 \\
\hline \multicolumn{2}{|c|}{ Resultados } \\
\hline Ninguno & $\begin{array}{l}\text { Los resultados se presentan de manera ordenada, en cohe- } \\
\text { rencia con los planteamientos iniciales. Se sugiere incluir } \\
\text { en el texto, la figura } 3 \text {, que se presenta al final, pero no se } \\
\text { describe en los hallazgos. }\end{array}$ \\
\hline \multicolumn{2}{|c|}{ Discusión } \\
\hline Ninguna & $\begin{array}{l}\text { La discusión es clara y apropiada, aporta al vacío de cono- } \\
\text { cimiento presentado al inicio del documento. }\end{array}$ \\
\hline \multicolumn{2}{|c|}{ Conclusiones } \\
\hline Ninguno & $\begin{array}{l}\text { Las conclusiones se presentan de manera concreta y } \\
\text { dejando clara la posibilidad de investigaciones futuras } \\
\text { para profundizar en el tema y brindas mayores aportes } \\
\text { en el tema. }\end{array}$ \\
\hline \multicolumn{2}{|c|}{ Referencias } \\
\hline Las referencias en su mayoría son mayores a 5 años. & $\begin{array}{l}\text { Se sugiere mejorar las referencias, de acuerdo con: } 1 \text {. In- } \\
\text { cluir en la lista de referencias, todas las que aparecen en } \\
\text { el texto y viceversa. } 2 \text {. Incluir referencias de los últimos } \\
\text { cinco años. }\end{array}$ \\
\hline
\end{tabular}


